Voix et Images

voixetimages

\title{
Jean Rivard ou l'art de réussir, de Robert Major
}

\section{David M. Hayne}

Volume 17, numéro 2 (50), hiver 1992

L’âge de la critique, 1920-1940

URI : https://id.erudit.org/iderudit/200965ar

DOI : https://doi.org/10.7202/200965ar

Aller au sommaire du numéro

\section{Éditeur(s)}

Université du Québec à Montréal

\section{ISSN}

0318-9201 (imprimé)

1705-933X (numérique)

Découvrir la revue

\section{Citer cet article}

Hayne, D. M. (1992). Jean Rivard ou l'art de réussir, de Robert Major. Voix et Images, 17(2), 320-324. https://doi.org/10.7202/200965ar d'utilisation que vous pouvez consulter en ligne.

https://apropos.erudit.org/fr/usagers/politique-dutilisation/ 


\section{Jean Rivard ou l'art de réussir, de Robert Major}

David M. Hayne, Université de Toronto

Le * stupide * XIX' siècle québécois, longtemps négligé, nous paraît aujourd'hui moins bête, grâce au progrès de la recherche depuis trente ans. Parmi les problèmes que ce siècle peu connu continue toutefois à nous poser, l'un des plus fondamentaux est celui de l'insertion du Québec d'autrefois dans la réalité nord-américaine et anglo-saxonne qui l'entourait. Le sujet avait été abordé de façon 
globale par Guildo Rousseau dans son ouvrage L'Image des États-Unis dans la littérature québécoise (1775-1930), lequel réunissait la documentation de base. Aujourd'hui, Robert Major nous propose pour sa part la relecture d'un "classique. du $\mathrm{XIX}^{\mathrm{e}}$ siècle québécois, le Jean Rivard d'Antoine Gérin-Lajoie ${ }^{1}$. Le but du critique est double: offrir une interprétation nouvelle et inédite d'un ouvrage dont le statut semblait acquis, et éclairer un aspect de la mentalité des *Canadiens. du siècle dernier.

L'introduction de son étude Jean Rivard ou l'art de réussir met en relief l'ambiguité idéologique du roman, qui manifeste une *profonde américanité - $(p, 16)$ tout en sauvegardant les apparences d'une doxa nationale conservatrice, catholique et agriculturiste: S'inspirant en partie de la sociocritique de Claude Duchet, Major justifie son choix du roman de Gérin-Lajoie: l'ouvrage, bien accueilli à son époque, quoique déconsidéré de nos jours, véhicule un contenu idéologique et utopique plus complexe qu'il ne paraît à première vue.

Le premier chapitre, "La genèse d'un discours", passe en revue les contacts américains et anglo-saxons qui ont influencé le jeune GérinLajoie: ses deux voyages aux États-Unis en 1844 et en 1851-1852, son apprentissage de la langue anglaise au collège de Nicolet, l'ascendant exercé sur le collégien par l'anglophile abbé Jean-Baptiste-Antoine Ferlan'd, et l'impact des conférences socio-économiques de son futur beau-père Étienne Parent. Robert Major souligne aussi l'apport probable des écrits américains de deux voyageurs français, Volney et Tocqueville: le premier fut cité longuement dans la première version (1864) de Jean Rivard économiste et le second semble avoir suggéré plusieurs des commentaires de Gérin-Lajoie sur l'indépendance d'esprit, l'ambition, et l'industrie de son héros fictif. D'autres écrivains américains que Gérin-Lajoie a sans doute connus, en particulier Franklin et Emerson, figurent dans un appendice à la fin du livre. Curieusement, Major n'émet aucune hypothèse, au cours de cette énumération systématique des influences américaines et anglo-saxonnes, sur les trois années que Gérin-Lajoie a passées entre février 1856 et juin 1859 dans un milieu anglo-saxon en pleine expansion commerciale, la ville de Toronto.

Le deuxième chapitre, "Paratexte et intrigue ", analyse minutieusement les titres, préfaces, avant-propos, notes et appendices des éditions pré-originale (1862-1864) et originale (1874) du roman, et examine les interventions du romancier dans son récit. Ces éléments paraet métatextuels sont particulièrement significatifs: Gérin-Lajoie communique par leur intermédiaire son intention d'offrir à des lecteurs 
sérieux une "monographie romancée. (Arthur Saint-Pierre) qui présente un défricheur de type américain susceptible de servir d'exemple à des Canadiens français réticents et casaniers. Major insiste sur le fait que Jean Rivard n'est pas un cultivateur: "Il est défricheur deux ans, économiste pendant une quinzaine d'années par la suite [...]", écrit-il (p. 73). Les vocables a fortune" et a richesse " reviennent souvent sous la plume de Gérin-Lajoie et les calculs de revenu tiennent une grande place dans le programme de son pionnier capitaliste.

Il est évident que cette interprétation du roman va à l'encontre de l'acception traditionnelle de l'œuvre: Camille Roy y voyait "le roman du colon. (Nouveaux Essais..., p. 84) èt l'opinion critique l'a suivi. Pourtant l'«Index et concordance de Jean Rivard. dressé par Hubert Larocque à l'Université d'Ottawa révèle que le terme de colon est peu usité dans le roman, et jamais pour désigner Jean Rivard lui-même. Ce dernier est un propriétaire indépendent et même prospère, tandis que le colon, pauvre et peu instruit, reçoit une terre agricole publique sujette à certaines conditions. Dans son troisième chapitre intitulé "Colonisation et agriculturisme", Robert Major fait ressortir cette distinction: Jean Rivard est un "producteur agricole" qui exploite une ferme comme source de revenu et se livre simultanément à des industries connexes (sucre d'érable, beurre, potasse). Les principaux porte-parole de l'idéologie agriculturiste dans le roman ne sont pas Jean Rivard et Pierre Gagnon, mais le citadin Gustave Charmenil (expédié un peu cavalièrement à la p. 81). et les curés Leblanc et Doucet; le romancier aurait lui-même retiré de l'édition originale des épigraphes qui faisaient l'éloge de l'agriculture (p. 138-139).

Le chapitre suivant traite de la "riche intertextualité. (p. 207) du roman. Il s'ouvre par une page un peu rapide (p. 149-150) sur la quasiinexistence de bibliothèques personnelles dans le Québec du XIX ${ }^{c}$ siècle. L'ouvrage d'Yvan Lamonde et Daniel Olivier, Les Bibliothèques personnelles au Québec... (Montréal, BNQ, 1983) nous signale au contraire la présence de bibliothèques importantes dans le cercle même de Gérin-Lajoie: 3000 volumes chez son frère Elzéar et 2200 volumes appartenant à son ami Raphaël Bellemare (Lamonde, p. 47, 115). Le cas de la bibliothèque de son ancien idole Louis-Joseph Papineau, dont Roger Le Moine a reconstitué le catalogue, est notoire. La collection de "quatre ou cinq cents volumes" réunie par l'économiste Jean Rivard est néanmoins impressionnante: elle atteste non seulement l'importance de la lecture dans la vie de Jean Rivard, mais aussi le rôle des livres dans la carrière du bibliothécaire Gérin-Lajoie. Parmi les ouvrages conservés par Jean Rivard, quatre sont mis en valeur dans le roman et Robert Major les analyse finement, en mettant l'accent sur la valeur exemplaire de 
Robinson Crusoé et de Napoléon $1^{\alpha}$. Cependant le critique ne semble pas connaître l'article de Claude Galarneau, "La légende napoléonienne au Québec. (dans Fernand Dumont et Yves Martin (directeurs), Imaginaire social et représentations collectives, Québec, P.U.L., 1982, p. 163174). Le chapitre se termine sur une analyse perspicace de la signification politique du culte de Napoléon vers 1862 : la fidélité de GérinLajoie le situe *nettement à gauche, du côté des libéraux et des anticléricaux * (p. 206), rapprochement que les lecteurs de l'époque n'auraient pas manqué de faire en lisant les appréciations de Jean Rivard signées par les libéraux Louis-Michel Darveau (p. 4) et Edmond Lareau (p. 5).

Le cinquième chapitre porte le titre parallèle d's Intertextualité II', mais il s'agit cette fois d'un thème litteraire universel, l'utopie. L'Utopieforschung étant plus ou moins inconnue au Québec, Robert Major se voit obligé de résumer l'état des études avant de préciser la-situation de Jean Rivard. Ni "paradis terrestre " ni "roman à thèse ", et encore moins création compensatoire d'un auteur frustré, Jean Rivard. serait plutôt une critique sociale présentée à l'aide des ressources normales du genre (forme épistolaire, disposition symétrique, narrateur étranger). La critique sociale vise le matérialisme, la frivolité, l'oisiveté, l'enseignement dispensé dans' les collèges, les mœurs électorales et la corruption politique, que le romancier voudrait remplacer par l'intelligence, la lecture, l'industrie, un enseignement réformé et l'honnêtèté. Comme dans toutes les utopies, la pédagogie joue un grand rôle dans celle de Gérin-Lajoie. «En un mot, conclut Robert Major, cette utopie est typiquement et profondément américaine, misant sur l'individualisme comme facteur de progrès collectif, valorisant la passion du gain comme source d'enrichissement communautaire: (p. 256)

Un bref sixième chapitre, "Républicanisme ou féodalité en Laurentie , revient sur deux questions que le critique veut éclairer: la paroisse modèle décrite dans le roman est-elle un * château-fort "fermé contre toute nouveauté et tout progrès matériel, et la société envisagée par le romancier est-elle une société hiérarchisée et féodale? À ces deux questions le critique oppose un non retentissant: la seule fermeture de la paroisse est son refus de la vénalité politique, tandis que sa seule hiérarchisation est celle fondée sur le succès et la fortune. Loin d'être un roman "bleu ", Jean Rivard serait un roman "mauve. (Bernard Proulx), pragmatique, réaliste, et orienté vers l'avenir nordaméricain.

La «Conclusion" résume le vrai sens de Jean Rivard. C'est que Gérin-Lajoie * invite ses compatriotes à changer leurs habitudes 
collectives suicidaires pour pouvoir maintenir leur identité nationale" (p. 290). C'est en assumant pleinement leur individualisme et leur américanité que les Canadiens français deviendront de véritables Français d'Amérique.

Jean Rivard ou l'art de réussir est une étude fort originale, adroitement organisée en dépit de quelques répétitions, et bien documentée. Le texte a été revu avec soin et les erreurs sont minimes (certaines références infrapaginales sont légèrement inexactes; le *Joseph-Gabriel Barthe- des pages 21, 25, 311 et 331 semble être un simple lapsus). L'on pourrait peut-être compléter l'excellente bibliographie en y ajoutant les travaux de Denis Monière, l'article de JeanCharles Falardeau dans le Dictionnaire biographique du Canada, tome XI, et les thèses de Vida M. Bruce (M.A., McMaster, 1972) et de Rosanna Furgiuele (Ph.D., Toronto, 1983).

Espérons enfin que la parution de cette étude, remarquable va encourager "une relecture analogue d'autres œuvres de l'époque* (p. 284).

1. . Robert Major, Jean Rivard ou l'ant de réussir: idéologies et utopie dans l'oeuvre d'Antoine Gérin-Lajoie, Sainte-Foy, Les Presses de l'Université Laval, coll. aVie des lettres québécoises :, $\mathrm{n}^{\circ}$ 30, 1991, $338 \mathrm{p}$. 